\title{
3 Research Square \\ Impact of thymic epithelial primary cilia on T cell development
}

Osamu Kutomi ( $\square$ okutomi@yamanashi.ac.jp)

University of Yamanashi

\section{Shigenori Nonaka}

National Institute for Basic Biology

Katsuto Hozumi

Tokai University

\section{Sen Takeda}

University of Yamanashi

\section{Research Article}

Keywords: primary cilia, T cell development, thymic epithelial cell (TEC)

Posted Date: April 26th, 2021

DOl: https://doi.org/10.21203/rs.3.rs-463087/v1

License: (c) (i) This work is licensed under a Creative Commons Attribution 4.0 International License. Read Full License 


\section{Abstract}

Primary cilia are ubiquitous hair-like organelles, usually projecting from the apical cell surface. They are essential for the organogenesis and homeostasis of various physiological functions, and their dysfunction leads to a plethora of human diseases. However, there are few reports on the role of primary cilia in the immune system; therefore, we focused on their role in the thymus that nurtures immature lymphocytes to full-fledged T cells. After detecting primary cilia on the thymic epithelial cell (TEC) expressed transforming growth factor $\beta$ (TGF- $\beta$ ) receptor in the basal body, we established a line of an intraflagellar transport protein 88 (Ift88) knockout mice lacking primary cilia in TECs (Ift88 TEC null mutant) to clarify their precise role in thymic organogenesis and T cell differentiation. The Ift88-TEC null mutant mice showed stunted cilia or lack of cilia in TECs. The intercellular contact between T cells and the "thymic synapse" of medullary TECs was slightly disorganized in Ift88-TEC null mutants. Notably, the CD4- and CD8-single positive thymocyte subsets increased significantly. The absence or disorganization of thymic cilia downregulated the TGF- $\beta$ signaling cascade, increasing the number of single positive thymocytes.

\section{Full Text}

Due to technical limitations, full-text HTML conversion of this manuscript could not be completed. However, the manuscript can be downloaded and accessed as a PDF.

\section{Figures}


A

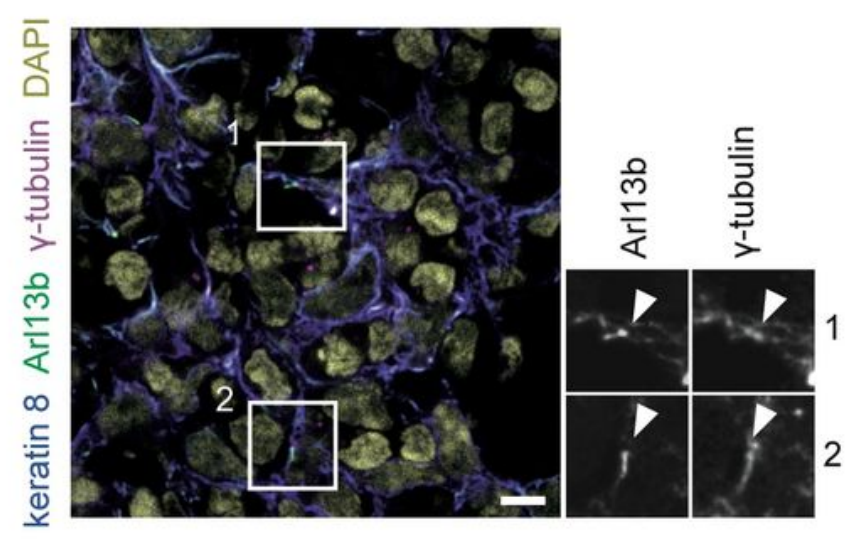

B

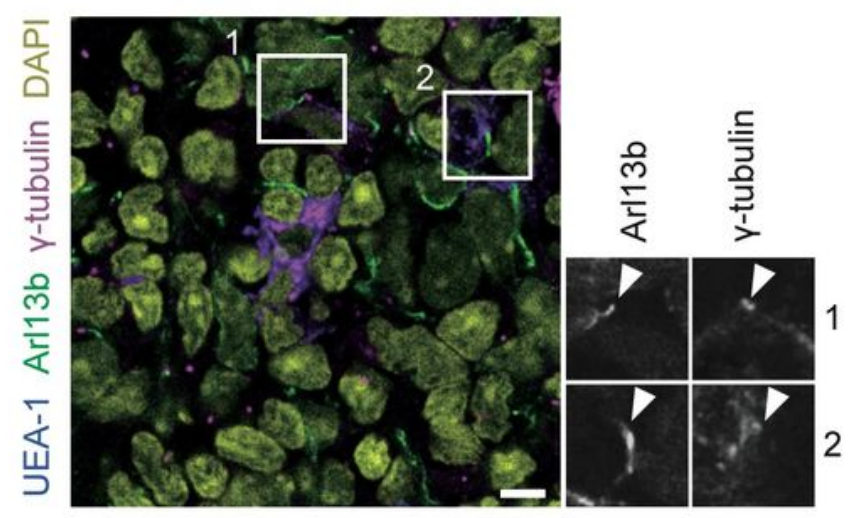

C

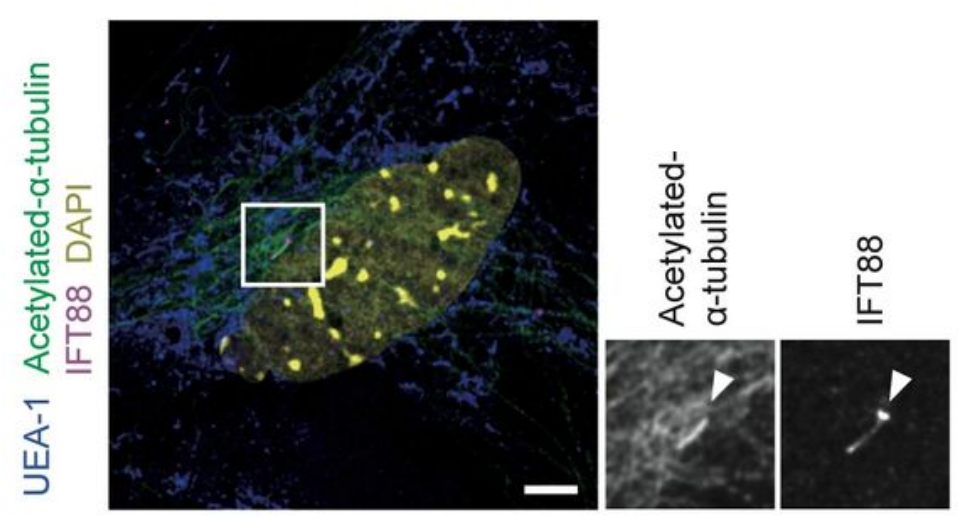

\section{D}

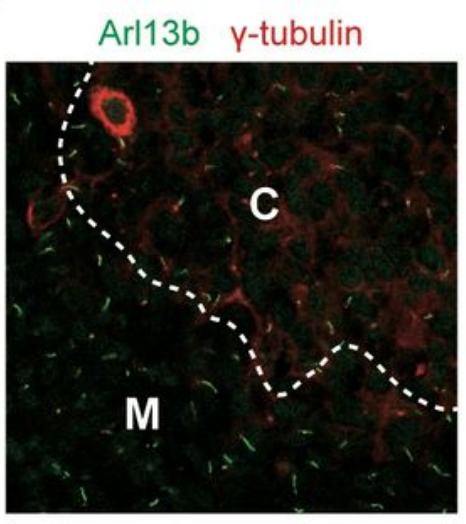

keratin 8 keratin 14
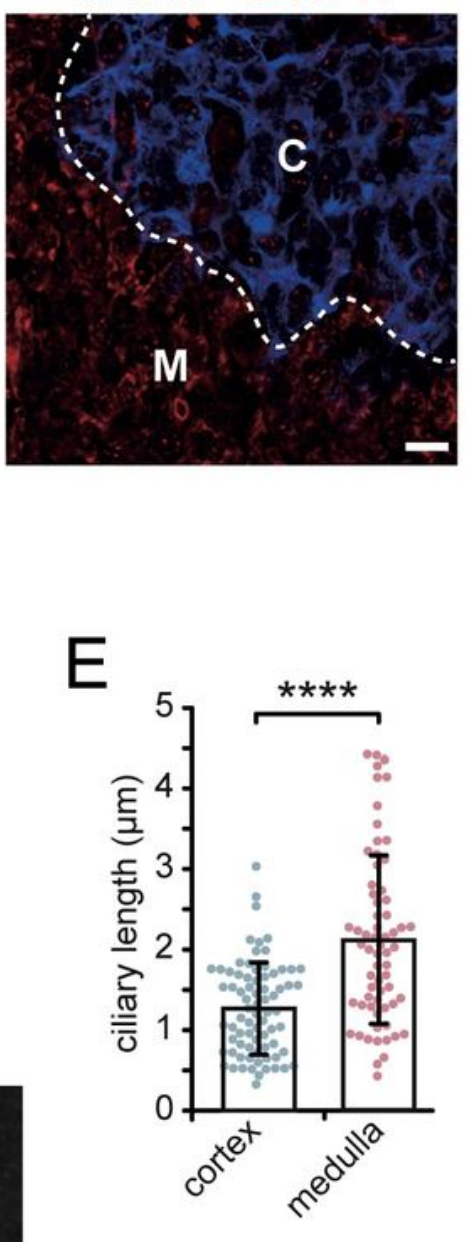

\section{Figure 1}

Immunohistochemical identification of primary thymus cilia. (A \& B) Fetal thymic organ culture (FTOC) from E15 B6 mice stained with anti-Arl13b (primary cilia marker), anti-y-tubulin (ciliary basal body marker), anti-keratin 8 (A, cTEC marker), UEA-1 (B, mTEC marker), and DAPI. White boxes in left panels enlarged and shown separately on right side to focus on cilia. Arrowheads indicate primary cilia projected from cTECs or mTECs. Scale bars: $5 \mu \mathrm{m}$. (C) Localization of alternative molecular primary cilia markers, 
acetylated-a-tubulin and IFT88 reveal a cilium in cultured mTECs. Scale bars: $5 \mu \mathrm{m}$. (D) FTOC treated with 2-dGuo and stained with anti-Arl13b and anti-y-tubulin antibodies. Cortex and medulla were discriminated by anti-keratin 8 (cTECs marker) and anti-keratin 14 (mTECs marker) antibodies. C: cortex; M: medulla. Scale bar: $10 \mu \mathrm{m}$. (E) Length of primary cilia determined by measuring segment path from ciliary base to tip visualized by anti-Arl13b antibody. Graphical summary of average length of primary cilia (mean \pm SD). Each plot represents individual data of cilium length ( $n=76$ and 65 for cortex and medulla, respectively). $\star \star \star \star p<0.0001$ (unpaired Student's t-test). FTOC: fetal thymus organ culture; DAPI: 4囚,6-diamidino-2phenylindole; cTEC: cortex thymic epithelial cell; mTEC: medulla thymic epithelial cell. 


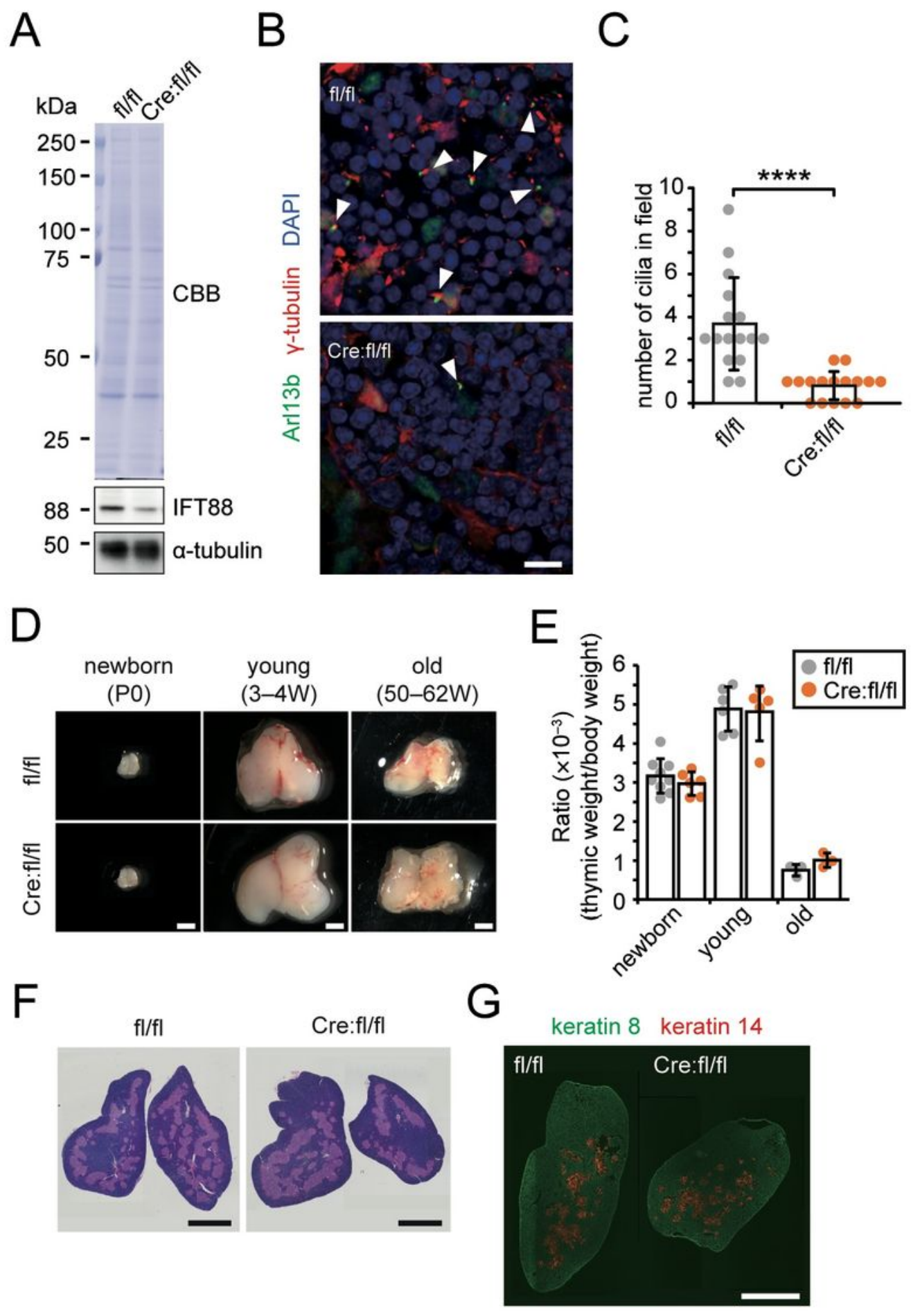

\section{Figure 2}

Characterization of Ift88fl/fl17 ::Foxn1-Cre conditional knockout mice (Ift88-TEC null mutants). (A) Western blotting analysis showing IFT88 thymic lysate expression in newborn mice. TEC expression has almost completely disappeared; however, there is a faint band at the height of IFT88 (arrowhead) due to continuation of expression in fibroblasts and thymocytes. (B) Newborn mice thymus probed with antiArl13b and anti-y-tubulin antibodies. Arrowheads indicate basal bodies reinforcing ciliary root. Scale bar: 
$10 \mu \mathrm{m}$. (C) Frequency of primary cilia (mean \pm SD) in newborn thymic tissue quantified from arbitrary microscopic fields $(105.369 \times 105.369 \mu \mathrm{m})$. Average number of cilia per field was significantly reduced $(\mathrm{n}$ $=16$ ) in Ift88-TEC null mutants (Cre:fl/fl) compared with IFT88fl/fl mice (fl/fl). ****p $<0.0001$ (unpaired Student's t-test). (D) Macroscopic thymus inspection in newborn, young, and old mice. Scale bar: $2 \mathrm{~mm}$. (E) Similar thymic/body weight ratio in controls and Ift88-TEC null mutant mice at all ages (mean \pm SD) $(n=3-9)$. (F) Histology of thymus from 4-week-old control and Ift88-TEC null mutant mice. Left panels show hematoxylin and eosin staining. (G) Immunohistochemical staining of sections from 4-week-old mice probed with anti-keratin 8 and 14 antibodies. Scale bars: $2 \mathrm{~mm}$. TEC: thymic epithelial cell. 
A

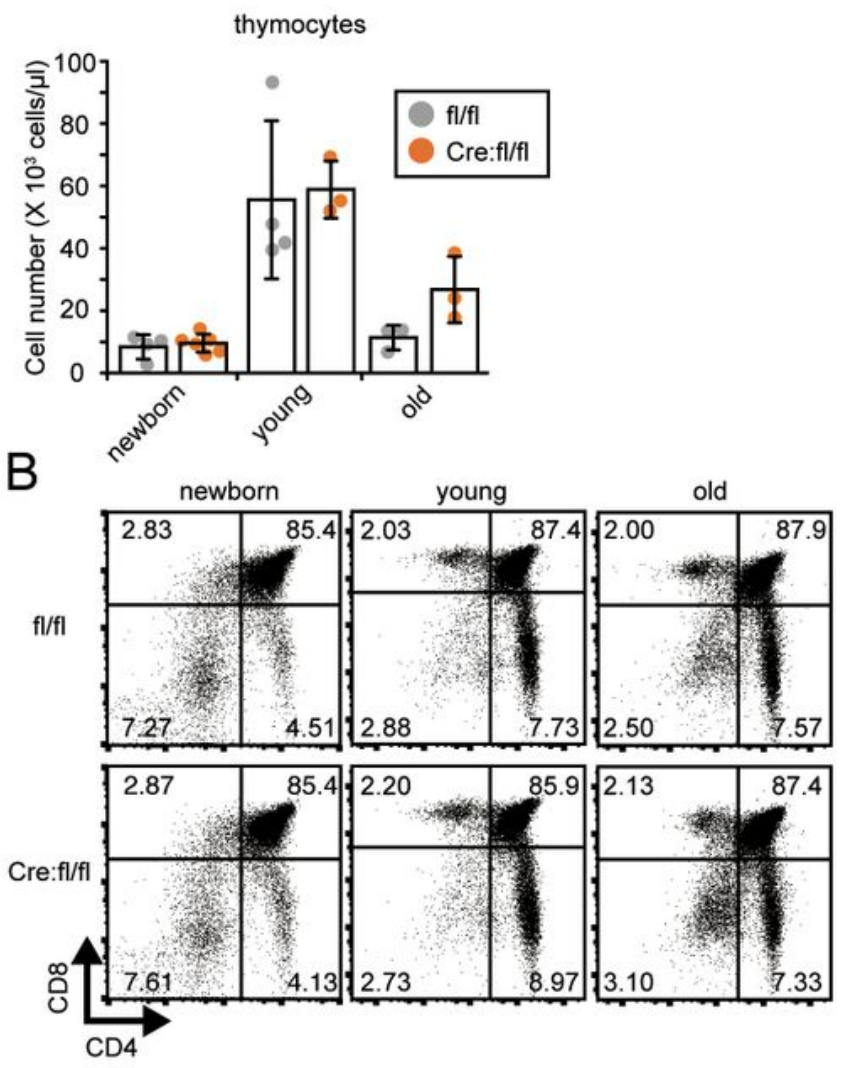

C
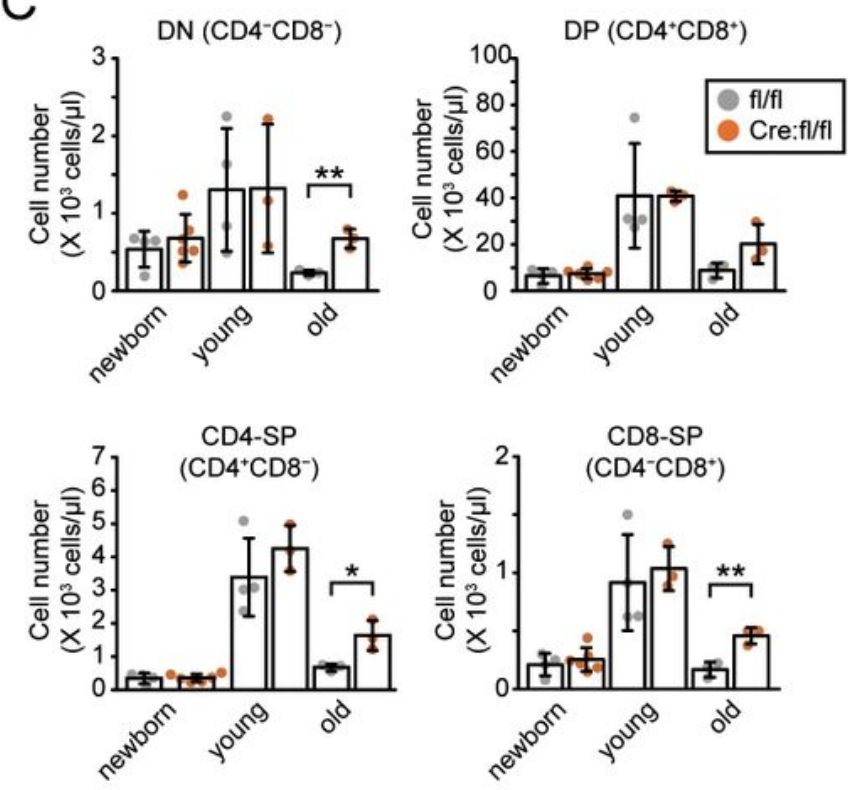

\section{Figure 3}

cells flow cytometry in Ift88-TEC null mutant mice at newborn, young, and old age (A) Cell count of thymocytes per thymic lobe. (B) T lymphocytes separated by either CD4 or/and CD8 expression at newborn, young, and old ages. Values in panels represent average ratio of each subset in each quadrant. (C) Graphical summary of panel (B) (mean \pm SD) showing cell counts for DN (CD4 -CD8-), DP 
D4+CD8+), CD4-SP (CD4+CD8-), and CD8-SP (CD4-CD8+ ) thymocytes. ${ }^{* \star} p<0.01,{ }^{\star} p<70.05$ (unpaired Student's t-test). TEC: thymic epithelial cell.
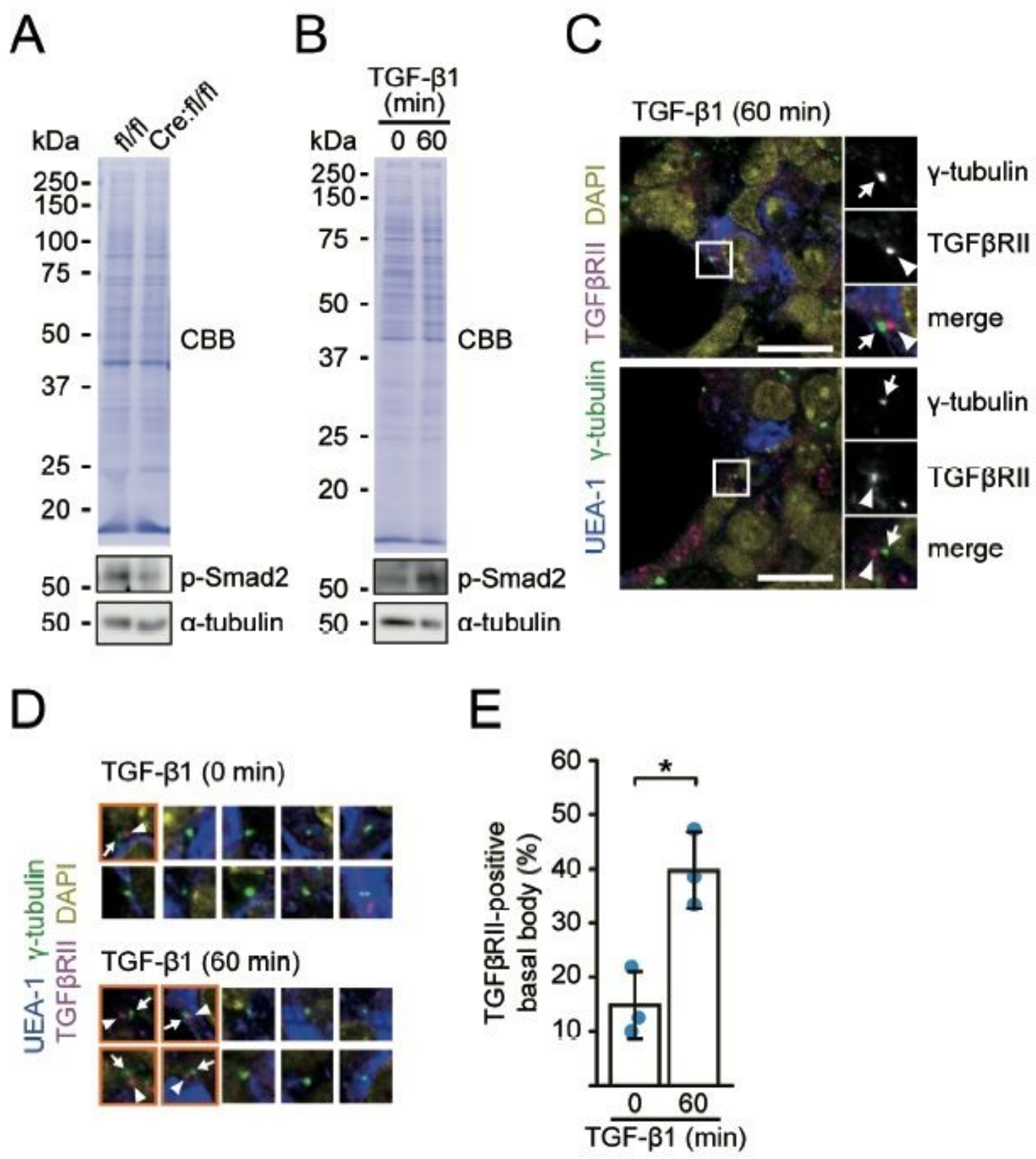

Figure 4

Primary cilia involved in controlling TGF- $\beta$ signaling in thymus. (A) Western blotting showing p-Smad2 in thymic lysate from newborn WT and Ift88 TEC null mice. (B) Western blotting showing Smad2 phosphorylation (p-Smad2) in FTOC treated with $2 \mathrm{ng} / \mathrm{ml}$ TGF- $\beta 1$ stimulation for 0 and $60 \mathrm{~min}$. (C) Typical images for localization of TGF $\beta$ RII in mTECs with TGF-1 $\beta 1$ stimulation for $60 \mathrm{~min}$. Arrows and arrowheads indicate $y$-tubulin and TGFßRII, respectively. Scale bars: $10 \mu \mathrm{m}$. (D) Galleries showing 
TGF $\beta$ RII adjacent to basal body (indicated by orange box) or basal body alone in mTECs. (E) Graphical representation of ratio of TGF $\beta R$ Il accumulation to basal body (mean \pm SD) in mTECs from FTOC treated with TGF- $\beta$ I stimulation for 0 or 60 min ( $n=3 ; 10-32$ basal bodies per section). ${ }^{*} p<0.05$ (unpaired Student's t-test). TEC: thymic epithelial cell; FTOC: fetal thymus organ culture; WT: wild type; TGF- $\beta$ : transforming growth factor $\beta$.

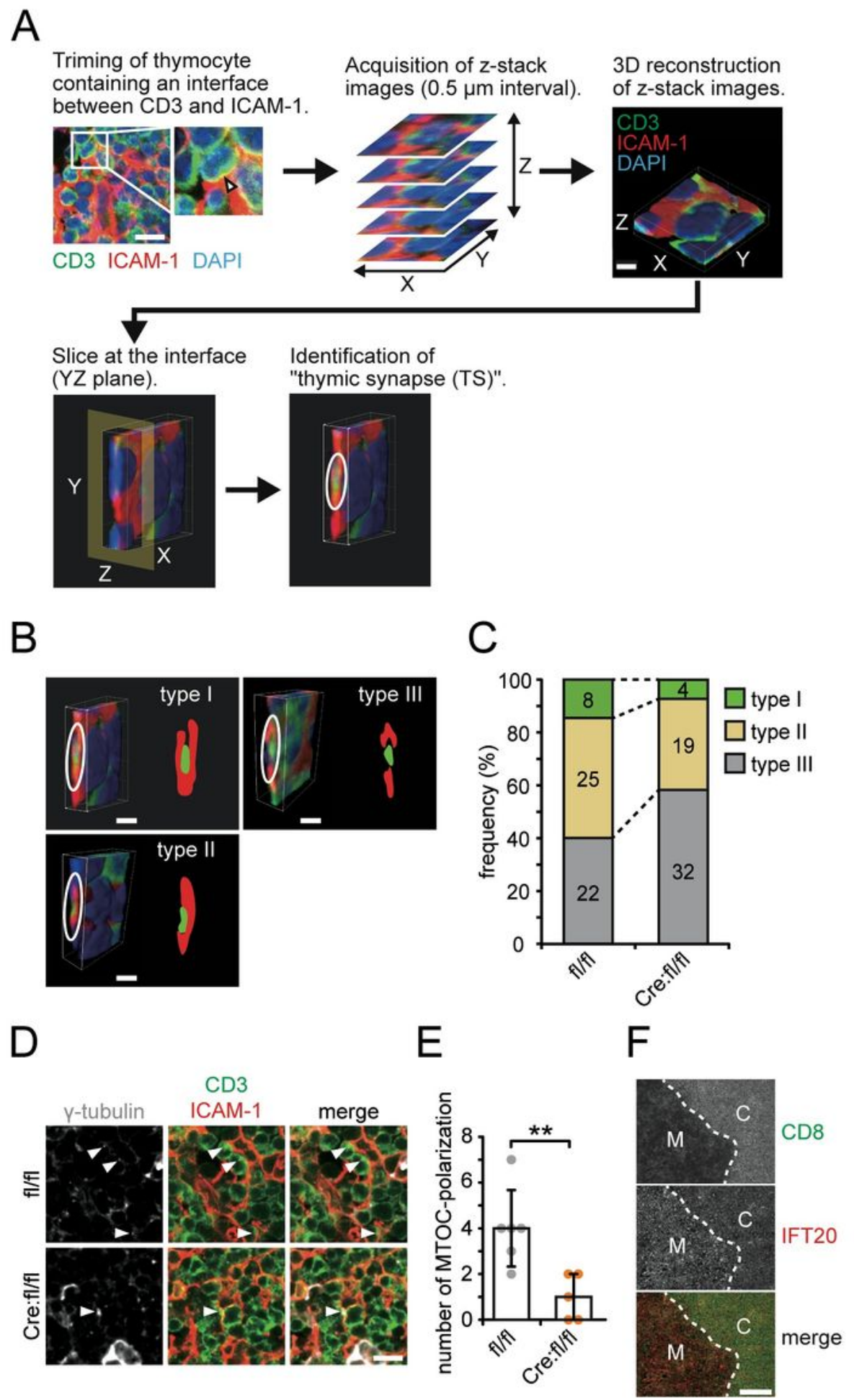

Figure 5 
Morphological identification of thymic synapse. (A) Workflow to reconstruct 3D image by volumerendering (blend mode) using Imaris viewer. TS identification by ICAM-1 signal (red) surrounding CD3 (green), positive area is indicated by white circles. Scale bars: $2 \mu \mathrm{m}$. (B) Catalog of three types of TS: typical ring-like-form (type I), quasi-ring form (type II), and fragmentation of ring (type III). Scale bars: 2 $\mu \mathrm{m}$. (C) Distribution of each TS subtype in controls $(n=55)$ and Ift88 TEC null mutant mice $(n=55)$. Each number within bars represents individual TS pattern frequency. (D) Polarization of MTOC to TS in newborn mice. Tissue sections stained with anti-y-tubulin (gray), anti- CD3 (green), and anti-ICAM-1 (red) antibodies. Arrowheads indicate y-tubulin pointing to TS. Scale bar: $10 \mu \mathrm{m}$. (E) Graphical summary of panel (D) showing MTOC polarization frequency to TS in newborn mice. Ift88 TEC null mutants show significantly reduced polarization ( $n=6$ and 5 for control and IFT88- cKO mice, respectively). ${ }^{\star \star} p<0.01$ (unpaired Student's t-test). (F) Expression of IFT20 in thymic tissue. Thymus from young mice stained with anti-IFT20 (red) and anti-CD8 (green) antibodies. CD8 and IFT20 expressed specifically in cortical and medullary thymocytes, respectively. Scale bar: $20 \mu \mathrm{m}$. C: cortex; M: medulla. TS: thymic synapse; TEC: thymic epithelial cell; MTOC: microtubule-organizing center; KO: knockout.

\section{Supplementary Files}

This is a list of supplementary files associated with this preprint. Click to download.

- KutomiSupplementaryData.pdf 